\title{
Numerical study of time-fractional hyperbolic partial differential equations
}

\author{
Saima Arshed \\ Department of Mathematics, University of the Punjab, Lahore 54590, Pakistan.
}

\begin{abstract}
In this study, a numerical scheme is developed for the solution of time-fractional hyperbolic partial differential equation. In the proposed scheme, cubic B-spline collocation is used for space discretization and time discretization is obtained by using central difference formula. Caputo fractional derivative is used for time-fractional derivative. The stability and convergence of the developed scheme, have also been proved. The numerical examples support the theoretical results. (C)2017 All rights reserved.
\end{abstract}

Keywords: Time-fractional hyperbolic equation, cubic B-spline, collocation method, convergence analysis, stability analysis. 2010 MSC: 35R11, 74H15.

\section{Introduction}

The idea of differentiation and integration to non-integer order has been studied in the fractional calculus. The subject fractional calculus is the generalized form of classical calculus. This subject is as old as conventional calculus, but researchers did not pay attention towards this subject, for so many years.

In recent decades, it has been observed by many mathematicians, scientists and researchers that fractional derivatives and integrals play a significant role in describing the properties of physical phenomena. Fractional derivatives and integrals efficiently describe the memory and hereditary properties of different processes and materials. It has further been observed that fractional models are more efficient and accurate than already developed classical models. The mathematical modeling of real world problems such as earthquake, traffic flow, fluid flow, signal processing, viscoelastic problems give rise to fractional partial differential equations (FPDEs).

Sometimes it is hard to calculate the exact solutions of fractional partial differential equations by using analytical methods. Due to this reason, mathematicians are motivated to develop numerical methods for the numerical solution of fractional PDEs. The numerical methods for solving fractional partial differential equations have become popular among the researchers in the last ten years. A variety of numerical methods have been used for finding the solution of FPDEs, such as the finite difference method, finite element method, finite volume method and the collocation method etc.

In this article, a numerical solution for time-fractional hyperbolic partial differential equation has been proposed.

Email address: saima .math@pu.edu.pk (Saima Arshed)

doi:10.22436/jmcs.017.01.05 
The considered equation has the following form

$$
\frac{\partial^{\alpha} u}{\partial t^{\alpha}}+a \frac{\partial u}{\partial t}+r u=\frac{\partial^{2} u}{\partial x^{2}}+f(x, t), \quad x \in \Omega=[0, L], \quad 0<t \leqslant T,
$$

with initial conditions

$$
u(x, 0)=g_{0}(x), \quad x \in \Omega, \frac{\partial u(x, 0)}{\partial t}=g_{1}(x)
$$

and boundary conditions

$$
u(0, t)=0, \quad u(L, t)=0, \quad t \in[0, T],
$$

where $1<\alpha<2$ is a parameter describing the order of the fractional time and $a, r$ are constants. $g_{0}(x)$ and $g_{1}(x)$ are given smooth functions and $f(x, t)$ is the source term.

The time-fractional derivative of order $\alpha$ has been considered in the Caputo form, defined as

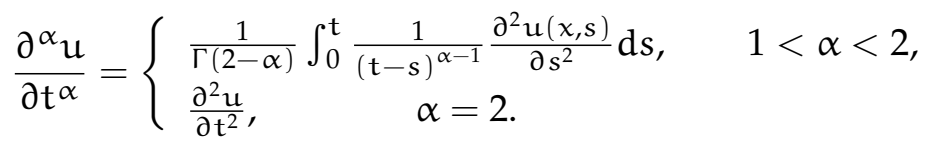

For $a>0, r>0,(1.1)$ is a fractional telegraph equation. The study of flow of electric signals in transmission line and wave propagation phenomena gives rise to telegraph equation. It is also used for modeling reaction diffusion equation.

For $a>0, r=0,(1.1)$ takes the following form

$$
\frac{\partial^{\alpha} u}{\partial t^{\alpha}}+a \frac{\partial u}{\partial t}=\frac{\partial^{2} u}{\partial x^{2}}+f(x, t),
$$

which represents a fractional damped wave equation.

For $\mathrm{a}=0, \mathrm{r}=0,(1.1)$ becomes

$$
\frac{\partial^{\alpha} u}{\partial t^{\alpha}}=\frac{\partial^{2} u}{\partial x^{2}}+f(x, t)
$$

which represents a fractional wave equation.

For $\mathrm{a}=0, \mathrm{r}>0,(1.1)$ becomes

$$
\frac{\partial^{\alpha} u}{\partial t^{\alpha}}+r u=\frac{\partial^{2} u}{\partial x^{2}}+f(x, t),
$$

which represents a fractional linear Klein-Gordon equation. The equation occurs in relativistic physics and is used to describe dispersive wave phenomena.

The numerical solution of partial differential equations with sub and super diffusion is proposed by Li et al. [16] . Wei et al. [28] proposed the numerical solution of fractional telegraph equation. Chen et al. [13] presented the approximate and exact solutions of the fractional diffusion-wave equation. Srivastava et al. [24] developed the method for solving telegraph equation. Sweilam and Assiri [25] presented finite difference scheme for the solution of nonlinear wave equation with fractional derivative. The numerical solution of fractional convection-diffusion equations is proposed by Zhang [29] by using finite difference method. Ding and Li [14] proposed a numerical algorithm for the fractional diffusion-wave equation. Siddiqi and Arshed [22] presented a numerical solution of convection-diffusion integro-differential equations with a weakly singular kernel. Tadjeran et al. [26] developed the solution of fractional diffusion equation. Liu et al. [19] provided the stability and convergence of the difference schemes for the fractional advection-diffusion equation. Sousa [23] proposed an approximation of the Caputo fractional derivative of order $1<\alpha \leqslant 2$. Liu et al. [18] developed a numerical method for solving the time fractional advectiondispersion equation. Zhang and Han [30] proposed a quasi-wavelet method for time-dependent fractional partial differential equation. Lynch et al. [20] proposed a numerical methods for the solution of partial differential equations of fractional order. In [8], Baskonus et al. used active control method for fractional 
order economic system. Lin and $\mathrm{Xu}$ [17] developed the approximate solution of time-fractional diffusion equation. Baskonus and Bulut [6] developed the numerical solutions of some fractional ordinary differential equations by fractional Adams-Bashforth-Moulton Method. Bulut et al. [11] proposed some new analytical solutions for the nonlinear time-fractional KdV-Burgers-Kuramoto equation. Baskonus et al. [5] used variational iteration method for the solutions of nonlinear fractional differential equations systems. Atangana [1-3] proposed the stability and a convergence analysis of fractional differential equations. Baskonus and Bulut [7] developed the complex analytical solutions for the fractional nonlinear double sinh-Poisson Equation. Bulut et al. [10] proposed modified trial equation method for fractional wave equation and time-fractional generalized Burgers equation. In [9], Bulut et al. developed the analytical solutions of some fractional ordinary differential equations by Sumudu Transform Method. In [4], Atangana et al. used the results from fractional calculus for the modeling of nonlinear wave motion. In [27], Demiray et al. used generalized Kudryashov method for solving time fractional differential equations. In [12], Bulut et al. developed exact solutions of time fractional $\mathrm{KdV}$ equations by using generalized Kudryashov method. In [21] finite difference approximations are used for fractional advection-dispersion flow equations. In [15] finite central difference/finite element approximations are applied for parabolic integro-differential equations.

The paper is divided into six sections. The cubic B-spline basis function is presented in Section 2. Section 3, presents the finite difference approximation for time discretization of the given problem. The stability and convergence of temporal discretization are established. Section 4, presents cubic B-spline collocation method for the space discretization. Approximate solutions that are discussed in Section 5, support the theoretical results. The concluding remarks are given in Section 6.

\section{Cubic B-spline}

The domain interval $[0,1]$ has been partitioned into $\mathrm{N}$ elements by having uniform step size $\mathrm{h}$ with knots $x_{i}, i=0,1,2, \ldots, N$ such that $\left\{0=x_{0}<x_{1}<x_{2}<\ldots<x_{N}=1\right\}, h=x_{i}-x_{i-1}, i=1,2, \ldots, N$.

The cubic B-spline basis function $B_{i}(x), i=-1,0, \ldots, N+1$ are defined as

$$
B_{i}(x)=\frac{1}{h^{3}} \begin{cases}\left(x-x_{i-2}\right)^{3}, & x \in\left[x_{i-2}, x_{i-1}\right], \\ h^{3}+3 h^{2}\left(x-x_{i-1}\right)+3 h\left(x-x_{i-1}\right)^{2}-3\left(x-x_{i-1}\right)^{3}, & x \in\left[x_{i-1}, x_{i}\right], \\ h^{3}+3 h^{2}\left(x_{i+1}-x\right)+3 h\left(x_{i+1}-x\right)^{2}-3\left(x_{i+1}-x\right)^{3}, & x \in\left[x_{i}, x_{i+1}\right] \\ \left(x_{i+2}-x\right)^{3}, & x \in\left[x_{i+1}, x_{i+2}\right] \\ 0, & \text { otherwise. }\end{cases}
$$

The values of successive derivatives $B_{i}^{(r)}(x), i=-1, \ldots, N+1, r=0,1,2$ at nodes, are listed in Table 1 .

Table 1: Coefficients of cubic B-spline and its derivatives at knots $x_{i}$.

\begin{tabular}{|c|c|c|c|c|c|c|}
\hline & $x_{i-2}$ & $x_{i-1}$ & $x_{i}$ & $x_{i+1}$ & $x_{i+2}$ & else \\
\hline$B_{i}(x)$ & 0 & 1 & 4 & 1 & 0 & 0 \\
$B_{i}^{\prime}(x)$ & 0 & $\frac{3}{h}$ & 0 & $\frac{-3}{h}$ & 0 & 0 \\
$B_{i}^{\prime \prime}(x)$ & 0 & $\frac{6}{h^{2}}$ & $\frac{-12}{h^{2}}$ & $\frac{6}{h^{2}}$ & 0 & 0 \\
\hline
\end{tabular}

The approximate solution can be written as a linear combination of cubic B-spline basis functions $\mathrm{B}_{\mathrm{i}}(\mathrm{x})$ as

$$
u^{n+1}(x)=\sum_{i=-1}^{N+1} c_{i}(t) B_{i}(x),
$$

where $c_{i}$ are time dependent unknown quantities to be computed from the boundary conditions and collocation form of the fractional differential equation. 


\section{Discretization in time}

The time-fractional derivative in (1.1) is defined in Caputo form. Let $t_{n}=n \Delta t, n=0,1,2, \ldots, K$, where $\Delta t=\frac{T}{K}$ is the temporal step size and $\mathrm{T}$ is the final time. Caputo fractional derivative is discretized by using the central difference approximation as

$$
\begin{aligned}
& \frac{\partial^{\alpha} u\left(x, t_{n+1}\right)}{\partial t^{\alpha}} \\
& =\frac{1}{\Gamma(2-\alpha)} \int_{0}^{t_{n+1}} \frac{\partial^{2} u(x, s)}{\partial s^{2}} \frac{d s}{\left(t_{n+1}-s\right)^{\alpha-1}}, \\
& =\frac{1}{\Gamma(2-\alpha)} \sum_{j=0}^{n} \int_{t_{j}}^{t_{j+1}} \frac{\partial^{2} u(x, s)}{\partial s^{2}} \frac{d s}{\left(t_{n+1}-s\right)^{\alpha-1}}, \\
& =\frac{1}{\Gamma(2-\alpha)} \sum_{j=0}^{n} \frac{u\left(x, t_{j+1}\right)-2 u\left(x, t_{j}\right)+u\left(x, t_{j-1}\right)}{\Delta t^{2}} \int_{t_{j}}^{t_{j+1}} \frac{d s}{\left(t_{n+1}-s\right)^{\alpha-1}}+r_{\Delta t}^{n+1}, \\
& =\frac{1}{\Gamma(2-\alpha)} \sum_{j=0}^{n} \frac{u\left(x, t_{j+1}\right)-2 u\left(x, t_{j}\right)+u\left(x, t_{j-1}\right)}{\Delta t^{2}} \int_{t_{n-j}}^{t_{n+1-j}} \frac{d \tau}{\tau^{\alpha-1}}+r_{\Delta t}^{n+1}, \\
& =\frac{1}{\Gamma(2-\alpha)} \sum_{j=0}^{n} \frac{u\left(x, t_{n+1-j}\right)-2 u\left(x, t_{n-j}\right)+u\left(x, t_{n-1-j}\right)}{\Delta t^{2}} \int_{t_{j}}^{t_{j+1}} \frac{d \tau}{\tau^{\alpha-1}}+r_{\Delta t}^{n+1}, \\
& =\frac{1}{\Gamma(3-\alpha)} \sum_{j=0}^{n} \frac{u\left(x, t_{n+1-j}\right)-2 u\left(x, t_{n-j}\right)+u\left(x, t_{n-1-j}\right)}{\Delta t^{\alpha}}\left((j+1)^{2-\alpha}-j^{2-\alpha}\right)+r_{\Delta t}^{n+1}, \\
& =\frac{1}{\Gamma(3-\alpha)} \sum_{j=0}^{n} b_{j} \frac{u\left(x, t_{n+1-j}\right)-2 u\left(x, t_{n-j}\right)+u\left(x, t_{n-1-j}\right)}{\Delta t^{\alpha}}+r_{\Delta t}^{n+1},
\end{aligned}
$$

where $b_{j}=(j+1)^{2-\alpha}-j^{2-\alpha}$ and $\tau=\left(t_{n+1}-s\right)$.

In particular, for $j=0$, denote $u^{-1}=u(x, 0)-\Delta t u^{(1)}(x, 0)=g_{0}(x)-\Delta t g_{1}(x)$.

Rewrite (3.1) in operator form as

$$
L_{t}^{\alpha} u\left(x, t_{n+1}\right):=\frac{1}{\Gamma(3-\alpha)} \sum_{j=0}^{n} b_{j} \frac{u\left(x, t_{n+1-j}\right)-2 u\left(x, t_{n-j}\right)+u\left(x, t_{n-1-j}\right)}{\Delta t^{\alpha}} .
$$

Then (3.1) leads to

$$
\frac{\partial^{\alpha} u}{\partial t^{\alpha}}=\frac{1}{\Gamma(2-\alpha)} \sum_{j=0}^{n} \int_{t_{j}}^{t_{j+1}} \frac{\partial^{2} u(x, s)}{\partial s^{2}} \frac{d s}{\left(t_{n+1}-s\right)^{\alpha-1}}=L_{t}^{\alpha} u\left(x, t_{n+1}\right)+r_{\Delta t}^{n+1} .
$$

The second order approximation of Caputo derivative as discussed in [23] is given as

$$
\left|r_{\Delta t}^{n+1}\right| \leqslant c \Delta t^{2}
$$

where $c$ is a constant.

By using $L_{t}^{\alpha} u\left(x, t_{n+1}\right)$ as an approximation of $\frac{\partial^{\alpha} u}{\partial t^{\alpha}}=\frac{1}{\Gamma(2-\alpha)} \int_{0}^{t_{n+1}} \frac{\partial^{2} u(x, s)}{\partial s^{2}} \frac{d s}{\left(t_{n+1}-s\right)^{\alpha-1}}$, the finite difference scheme for (1.1) has the form

$$
L_{t}^{\alpha} u\left(x, t_{n+1}\right)+a \frac{\partial u\left(x, t_{n+1}\right)}{\partial t}+r u\left(x, t_{n+1}\right)=\frac{\partial^{2} u\left(x, t_{n+1}\right)}{\partial x^{2}}+f\left(x, t_{n+1}\right) .
$$

By applying the $\theta$-weighted scheme to the space derivative at two adjacent time levels, the above equation takes the following form

$$
\mathrm{L}_{\mathrm{t}}^{\alpha} \mathrm{u}\left(\mathrm{x}, \mathrm{t}_{\mathrm{n}+1}\right)+\mathrm{a}\left(\frac{\mathrm{u}\left(\mathrm{x}, \mathrm{t}_{\mathrm{n}+1}\right)-\mathrm{u}\left(\mathrm{x}, \mathrm{t}_{\mathrm{n}-1}\right)}{2 \Delta \mathrm{t}}\right)+\theta\left\{\mathrm{ru}\left(\mathrm{x}, \mathrm{t}_{\mathrm{n}+1}\right)\right\}+(1-\theta)\left\{\mathrm{ru}\left(\mathrm{x}, \mathrm{t}_{\mathrm{n}}\right)\right\}
$$




$$
=\theta\left\{\frac{\partial^{2} u\left(x, t_{n+1}\right)}{\partial x^{2}}\right\}+(1-\theta)\left\{\frac{\partial^{2} u\left(x, t_{n}\right)}{\partial x^{2}}\right\}+f\left(x, t_{n+1}\right) .
$$

By taking $\theta=\frac{1}{2}$, we get

$$
\begin{gathered}
L_{t}^{\alpha} u\left(x, t_{n+1}\right)+a\left(\frac{u\left(x, t_{n+1}\right)-u\left(x, t_{n-1}\right)}{2 \Delta t}\right)+r\left(\frac{u\left(x, t_{n+1}\right)+u\left(x, t_{n}\right)}{2}\right) \\
=\left(\frac{u_{x x}\left(x, t_{n+1}\right)+u_{x x}\left(x, t_{n}\right)}{2}\right)+f\left(x, t_{n+1}\right) .
\end{gathered}
$$

By inserting the value of $L_{t}^{\alpha} u\left(x, t_{n+1}\right)$, we have

$$
\begin{gathered}
\frac{1}{\Gamma(3-\alpha)} \sum_{j=0}^{n} b_{j} \frac{u\left(x, t_{n+1-j}\right)-2 u\left(x, t_{n-j}\right)+u\left(x, t_{n-1-j}\right)}{\Delta t^{\alpha}}+a\left(\frac{u\left(x, t_{n+1}\right)-u\left(x, t_{n-1}\right)}{2 \Delta t}\right) \\
+r\left(\frac{u\left(x, t_{n+1}\right)+u\left(x, t_{n}\right)}{2}\right) \\
=\left(\frac{u_{x x}\left(x, t_{n+1}\right)+u_{x x}\left(x, t_{n}\right)}{2}\right)+f\left(x, t_{n+1}\right) .
\end{gathered}
$$

After simplification, the above scheme takes the following form

$$
\begin{aligned}
\left(1+\frac{a \alpha_{0}}{2 \Delta t}+\frac{r \alpha_{0}}{2}\right) u^{n+1}-\frac{\alpha_{0}}{2} u_{x x}^{n+1}= & -b_{n} u^{-1}+\left(2 b_{n}-b_{n-1}\right) u^{0} \\
& +\sum_{j=1}^{n-1}\left(-b_{j-1}+2 b_{j}-b_{j+1}\right) u^{n-j}+\left(2 b_{0}-b_{1}\right) u^{n} \\
& -\frac{r \alpha_{0}}{2} u^{n}+\frac{\alpha_{0}}{2} u_{x x}^{n}+\frac{a \alpha_{0}}{2 \Delta t} u^{n-1}+\alpha_{0} f^{n+1}, \quad n \geqslant 1,
\end{aligned}
$$

where $u^{n+1}(x)=u\left(x, t_{n+1}\right)$ and $\alpha_{0}=\Gamma(3-\alpha) \Delta t^{\alpha}$.

For $n=1$, the above scheme (3.5) becomes

$$
\begin{aligned}
\left(1+\frac{a \alpha_{0}}{2 \Delta t}+\frac{r \alpha_{0}}{2}\right) u^{2}-\frac{\alpha_{0}}{2} u_{x x}^{2}= & -b_{1} u^{-1}+\left(2 b_{1}-b_{0}\right) u^{0}+\left(2 b_{0}-b_{1}\right) u^{1}+\frac{a \alpha_{0}}{2 \Delta t} u^{0} \\
& +\frac{\alpha_{0}}{2} u_{x x}^{1}-\frac{r \alpha_{0}}{2} u^{1}+\alpha_{0} f^{2}
\end{aligned}
$$

For $n=0$, the above scheme can be rewritten as

$$
\begin{aligned}
\left(1+\frac{a \alpha_{0}}{2 \Delta t}+\frac{\alpha_{0} r}{2}\right) u^{1}-\frac{\alpha_{0}}{2} u_{x x}^{1}= & \left(-1+\frac{a \alpha_{0}}{2 \Delta t}\right) u^{-1}+\left(2-\frac{\alpha_{0} r}{2}\right) u^{0} \\
& +\frac{\alpha_{0}}{2} u_{x x}^{0}+\alpha_{0} f^{1}
\end{aligned}
$$

Equations (3.7) and (3.6), with the boundary conditions

$$
u^{n+1}(0)=u^{n+1}(L)=0, \quad n \geqslant 0,
$$

and initial conditions

$$
\begin{array}{r}
u(x, 0)=g_{0}(x), \quad x \in \Omega, \\
\frac{\partial u(x, 0)}{\partial t}=g_{1}(x),
\end{array}
$$

represent the time-discrete problem. 
The error term $r^{n+1}$ is defined as

$$
r^{n+1}:=\left(\frac{\partial^{\alpha} u\left(x, t_{n+1}\right)}{\partial t^{\alpha}}-L_{t}^{\alpha} u\left(x, t_{n+1}\right)\right) .
$$

By using (3.2),

$$
\left|r^{n+1}\right|=\left|r_{\Delta t}^{n+1}\right| \leqslant c \Delta t^{2}
$$

To check the stability and convergence of the time-discrete problem, the inner product and norms are defined as

$$
\begin{aligned}
& H_{0}^{1}(\Omega)=\left\{f \in H^{1}(\Omega),\left.f\right|_{\partial \Omega}=0\right\}, \\
& (f, g)_{1}=(f, g)+\left(\frac{\partial f}{\partial x}, \frac{\partial g}{\partial x}\right), \\
& \|f\|_{0}=(f, f)^{1 / 2}, \quad\|f\|_{1}=(f, f)_{1}^{1 / 2} .
\end{aligned}
$$

In this paper, it is preferred to apply $\|\cdot\|_{1}$ instead of $\mathrm{H}^{1}$-norm, defined as

$$
\|f\|_{1}=\left(\|f\|_{0}^{2}+A\left\|\frac{\partial f}{\partial x}\right\|_{0}^{2}\right)^{1 / 2},
$$

where

$$
A=\frac{\alpha_{0}}{2+\beta_{0}+r \alpha_{0}}, \quad \beta_{0}=\frac{a \alpha_{0}}{\Delta t} .
$$

The weak formulation of (3.7) and (3.6) has the following form, i.e., by finding $u^{n+1} \in H_{0}^{1}(\Omega)$, such that for all $v \in \mathrm{H}_{0}^{1}(\Omega)$,

$$
\begin{aligned}
\left(u^{1}, v\right)-A\left(u_{x x}^{1}, v\right)= & (-2 B+C)\left(u^{-1}, v\right)+(4 B-r A)\left(u^{0}, v\right) \\
& +A\left(u_{x x}^{0}, v\right)+2 A\left(f^{1}, v\right),
\end{aligned}
$$

and

$$
\begin{aligned}
\left(u^{n+1}, v\right)-A\left(u_{x x}^{n+1}, v\right)= & -2 B b_{n}\left(u^{-1}, v\right)+2 B\left(2 b_{n}-b_{n-1}\right)\left(u^{0}, v\right) \\
& +2 B \sum_{j=1}^{n-1}\left(-b_{j-1}+2 b_{j}-b_{j+1}\right)\left(u^{n-j}, v\right) \\
& +2 B\left(2-b_{1}\right)\left(u^{n}, v\right)-r A\left(u^{n}, v\right) \\
& +A\left(u_{x x}^{n}, v\right)+C\left(u^{n-1}, v\right)+2 A\left(f^{n+1}, v\right),
\end{aligned}
$$

where

$$
A=\frac{\alpha_{0}}{2+\beta_{0}+r \alpha_{0}}, \quad B=\frac{1}{2+\beta_{0}+r \alpha_{0}}, \quad C=\frac{\beta_{0}}{2+\beta_{0}+r \alpha_{0}} .
$$

\section{Discrete Gronwall Inequality}

If the sequences $\left\{a_{j}\right\}$ and $\left\{z_{j}\right\}, j=1,2, \ldots, n$, satisfy inequality

$$
z_{j} \leqslant\left(\sum_{i=1}^{j-1} a_{i} z_{i}+b\right), \quad j=1,2, \ldots, n,
$$

where $a_{j} \geqslant 0, b \geqslant 0$, then the inequality

$$
z_{j} \leqslant b \cdot \exp \left(\sum_{i=1}^{j-1} a_{i}\right), \quad j=1,2, \ldots, n .
$$




\section{Stability and convergence analysis}

The stability of time-discrete problem is discussed in the following theorem.

Theorem 4.1. The proposed time-discrete scheme (3.6) is stable, if for all $\Delta \mathrm{t}>0$, the following relation holds

$$
\left\|u^{n+1}\right\|_{1} \leqslant D\left(\left\|g_{0}\right\|_{0}+\Delta t \mid g_{1} \|_{0}\right), \quad n=0,1,2, \ldots, K-1,
$$

where $\|.\|_{1}$ is defined in (3.9).

Proof. For $n=0$, let $v=u^{1}$ in (3.10), it can be written as

$$
\begin{aligned}
\left(u^{1}, u^{1}\right)-A\left(u_{x x}^{1}, u^{1}\right)= & (-2 B+C)\left(u^{-1}, u^{1}\right)+(4 B-r A)\left(u^{0}, u^{1}\right) \\
& +A\left(u_{x x}^{0}, u^{1}\right) .
\end{aligned}
$$

By using integration by parts and applying the boundary conditions, the above equation becomes

$$
\left(u^{1}, u^{1}\right)+A\left(\frac{\partial u^{1}}{\partial x}, \frac{\partial u^{1}}{\partial x}\right)=(-2 B+C)\left(u^{-1}, u^{1}\right)+(4 B-r A)\left(u^{0}, u^{1}\right)-A\left(u_{x}^{0}, u_{x}^{1}\right) .
$$

By using

$$
\|v\|_{0} \leqslant\|v\|_{1}, \quad\left\|\frac{\partial v}{\partial x}\right\|_{0} \leqslant \sqrt{\frac{1}{A}}\|v\|_{1},
$$

and Schwarz inequality, the above equation takes the following form

$$
\begin{aligned}
\left\|u^{1}\right\|_{1}^{2} \leqslant & 2 B\left\|u^{-1}\right\|_{0}\left\|u^{1}\right\|_{1}+C\left\|u^{-1}\right\|_{0}\left\|u^{1}\right\|_{1}+4 B\left\|u^{0}\right\|_{0}\left\|u^{1}\right\|_{1} \\
& +\left\|u^{0}\right\|_{0}\left\|u^{1}\right\|_{1}+r A\left\|u^{0}\right\|_{0}\left\|u^{1}\right\|_{1}, \\
\left\|u^{1}\right\|_{1} \leqslant & (2 B+C)\left\|u^{-1}\right\|_{0}+(2+(2 B-C))\left\|u^{0}\right\|_{0},
\end{aligned}
$$

since $(2 \mathrm{~B} \pm \mathrm{C}) \leqslant 1$, the above equation can be rewritten as

$$
\left\|\mathrm{u}^{1}\right\|_{1} \leqslant\left\|\mathrm{u}^{-1}\right\|_{0}+3\left\|\mathrm{u}^{0}\right\|_{0} .
$$

By using the inequality

$$
\left\|u^{-1}\right\| \leqslant\left(\left\|g_{0}\right\|_{0}+\Delta t\left\|g_{1}\right\|_{0}\right)
$$

(4.3) becomes

$$
\left\|u^{1}\right\|_{1} \leqslant \mathrm{D}\left(\left\|g_{0}\right\|_{0}+\Delta t\left\|g_{1}\right\|_{0}\right)
$$

Assume that the above relation holds for $v=u^{j}$, i.e.,

$$
\left\|u^{j}\right\|_{1} \leqslant D\left(\left\|g_{0}\right\|_{0}+\Delta t\left\|g_{1}\right\|_{0}\right), \quad j=2,3, \ldots, n .
$$

For $v=u^{n+1}$ in (3.11), we have

$$
\begin{aligned}
\left(u^{n+1}, u^{n+1}\right)-A\left(\frac{\partial^{2} u^{n+1}}{\partial x^{2}}, u^{n+1}\right)= & -2 B b_{n}\left(u^{-1}, u^{n+1}\right)+2 B\left(2 b_{n}-b_{n-1}\right)\left(u^{0}, u^{n+1}\right) \\
& +2 B \sum_{j=1}^{n-1}\left(-b_{j-1}+2 b_{j}-b_{j+1}\right)\left(u^{n-j}, u^{n+1}\right) \\
& +2 B\left(2-b_{1}\right)\left(u^{n}, u^{n+1}\right)-r A\left(u^{n}, u^{n+1}\right) \\
& +A\left(\frac{\partial^{2} u^{n}}{\partial x^{2}}, u^{n+1}\right)+C\left(u^{n-1}, u^{n+1}\right) .
\end{aligned}
$$

By using integration by parts and applying boundary conditions, the above equation takes the following 
form

$$
\begin{aligned}
\left(u^{n+1}, u^{n+1}\right)+A\left(\frac{\partial u^{n+1}}{\partial x}, \frac{\partial u^{n+1}}{\partial x}\right)= & -2 B b_{n}\left(u^{-1}, u^{n+1}\right)+2 B\left(2 b_{n}-b_{n-1}\right)\left(u^{0}, u^{n+1}\right) \\
& +2 B \sum_{j=1}^{n-1}\left(-b_{j-1}+2 b_{j}-b_{j+1}\right)\left(u^{n-j}, u^{n+1}\right) \\
& +2 B\left(2-b_{1}\right)\left(u^{n}, u^{n+1}\right) \\
& +C\left(u^{n-1}, u^{n+1}\right)-A\left(\frac{\partial u^{n}}{\partial x}, \frac{\partial u^{n+1}}{\partial x}\right)-r A\left(u^{n}, u^{n+1}\right) .
\end{aligned}
$$

By using Schwartz inequality, it can be written as

$$
\begin{aligned}
\left\|u^{n+1}\right\|_{1}^{2} \leqslant & 2 B b_{n}\left\|u^{-1}\right\|_{0}\left\|u^{n+1}\right\|_{1}+2 B\left(2 b_{n}-b_{n-1}\right)\left\|u^{0}\right\|_{0}\left\|u^{n+1}\right\|_{1} \\
& +2 B \sum_{j=1}^{n-1}\left(-b_{j-1}+2 b_{j}-b_{j+1}\right)\left\|u^{n-j}\right\|_{1}\left\|u^{n+1}\right\|_{1}+2 B\left(2-b_{1}\right)\left\|u^{n}\right\|_{1}\left\|u^{n+1}\right\|_{1} \\
& +C\left\|u^{n-1}\right\|_{1}\left\|u^{n+1}\right\|_{1}+\left\|u^{n}\right\|_{1}\left\|u^{n+1}\right\|_{0}+r A\left\|u^{n}\right\|_{1}\left\|u^{n+1}\right\|_{1} .
\end{aligned}
$$

By using induction hypothesis and discrete Gronwall inequality (3.12), we have

$$
\begin{aligned}
\left\|\mathrm{u}^{\mathrm{n}+1}\right\|_{1} & \leqslant\left(\left\|g_{0}\right\|_{0}+\Delta \mathrm{t}\left\|g_{1}\right\|_{0}\right) \exp \left(2 \mathrm{~B}\left(1+\mathrm{b}_{\mathrm{n}-1}-\mathrm{b}_{\mathrm{n}}\right)+\mathrm{C}+1+\mathrm{rA}\right) \\
& \leqslant \mathrm{D}\left(\left\|g_{0}\right\|_{0}+\Delta \mathrm{t}\left\|g_{1}\right\|_{0}\right) .
\end{aligned}
$$

Theorem 4.2. The numerical solution obtained by the proposed method converges to the exact solution, if the following relation holds

$$
\left\|u\left(t_{n}\right)-u^{n}\right\|_{1} \leqslant E \Delta t^{2}, \quad n=1,2, \ldots, K .
$$

Proof. Let the error term is defined as $e^{n}=u\left(x, t_{n}\right)-u^{n}(x)$, for $n=1$, the error equation can be written as

$$
\left(e^{1}, v\right)+A\left(\frac{\partial e^{1}}{\partial x}, \frac{\partial v}{\partial x}\right)=(-2 B+C)\left(e^{-1}, v\right)+(4 B-r A)\left(e^{0}, v\right)-A\left(\frac{\partial e^{0}}{\partial x}, \frac{\partial v}{\partial x}\right)+\left(r^{1}, v\right)
$$

for all $v \in \mathrm{H}_{0}^{1}(\Omega)$.

Let $v=e^{1}$, noting $e^{0}=0$ yields

$$
\begin{gathered}
\left(e^{1}, e^{1}\right)+A\left(\frac{\partial e^{1}}{\partial x}, \frac{\partial e^{1}}{\partial x}\right)=(-2 B+C)\left(e^{-1}, e^{1}\right)+\left(r^{1}, e^{1}\right), \quad \forall v \in H_{0}^{1}(\Omega), \\
\left\|e^{1}\right\|_{1}^{2} \leqslant(2 B+C)\left\|e^{-1}\right\|_{0}\left\|e^{1}\right\|_{1}+\left\|r^{1}\right\|_{0}\left\|e^{1}\right\|_{1}, \quad \forall v \in \mathrm{H}_{0}^{1}(\Omega), \\
\left\|e^{1}\right\|_{1} \leqslant(2 B+C)\left\|e^{-1}\right\|_{0}+\left\|r^{1}\right\|_{0}, \quad \forall v \in \mathrm{H}_{0}^{1}(\Omega) .
\end{gathered}
$$

Since $(2 B+C)<1$ and $\left\|e^{-1}\right\| \leqslant \Delta t^{2}$, this together with (3.8), gives

$$
\left\|u\left(t_{1}\right)-u^{1}\right\|_{1} \leqslant E \Delta t^{2}=E \Delta t^{2} .
$$

Hence (4.6) is proved for the case $n=1$.

For inductive part, suppose (4.6) holds for $n=1,2,3, \ldots$, s, i.e.,

$$
\left\|u\left(t_{n}\right)-u^{n}\right\|_{1} \leqslant E \Delta t^{2}
$$


To prove the lemma for $n=s+1$, equations (1.1) and (3.11) are used and the error equation can be written, for all $v \in \mathrm{H}_{0}^{2}(\Omega)$, as

$$
\begin{aligned}
\left(e^{n+1}, e^{n+1}\right)+A\left(\frac{\partial e^{n+1}}{\partial x}, \frac{\partial e^{n+1}}{\partial x}\right)= & -2 B b_{n}\left(e^{-1}, e^{n+1}\right)+2 B\left(2 b_{n}-b_{n-1}\right)\left(e^{0}, e^{n+1}\right) \\
& +2 B \sum_{j=1}^{n-1}\left(-b_{j-1}+2 b_{j}-b_{j+1}\right)\left(e^{n-j}, e^{n+1}\right) \\
& +2 B\left(2-b_{1}\right)\left(e^{n}, e^{n+1}\right)-A\left(\frac{\partial e^{n}}{\partial x}, \frac{\partial e^{n+1}}{\partial x}\right)-r A\left(e^{n}, e^{n+1}\right) \\
& +C\left(e^{n-1}, e^{n+1}\right)+\left(r^{n+1}, e^{n+1}\right), \\
\left\|e^{n+1}\right\|_{1} \leqslant 2 B b_{n}\left\|e^{-1}\right\|_{0}+ & 2 B \sum_{j=1}^{n-1}\left(-b_{j-1}+2 b_{j}-b_{j+1}\right)\left\|e^{n-j}\right\|_{0} \\
& +2 B\left(2-b_{1}\right)\left\|e^{n}\right\|_{0}+\left\|e^{n}\right\|_{0}+r A\left\|e^{n}\right\|_{0}+C\left\|e^{n-1}\right\|_{0}+\left\|r^{n+1}\right\|_{0} .
\end{aligned}
$$

By using induction hypothesis and discrete Gronwall inequality (3.12), we have

$$
\begin{aligned}
&\left\|e^{n+1}\right\|_{1} \leqslant\left(2 B b_{n}\left\|e^{-1}\right\|_{0}+\left\|r^{n+1}\right\|_{0}\right) \\
& \times \exp \left(2 B \sum_{j=1}^{n-1}\left(-b_{j-1}+2 b_{j}-b_{j+1}\right)+2 B\left(2-b_{1}\right)+1+r A+C\right) \\
& \leqslant\left(2 B b_{n} \Delta t^{2}+\Delta t^{2}\right) \exp \left(2 B\left(1+b_{n-1}-b_{n}\right)+C+1+r A\right), \\
&\left.\| u\left(t_{n+1}\right)-u^{n+1}\right) \|_{1} \leqslant E \Delta t^{2},
\end{aligned}
$$

where $E$ is a constant.

\section{Space discretization using collocation method}

Cubic B-spline collocation method is used for spatial discretization. Substituting (2.1) into (3.6), we have the following equation

$$
\begin{aligned}
(1+ & \left.\frac{a \alpha_{0}}{2 \Delta t}+\frac{r \alpha_{0}}{2}\right)\left(c_{i-1}^{n+1}+4 c_{i}^{n+1}+c_{i+1}^{n+1}\right)-\frac{\alpha_{0}}{2} \frac{6}{h^{2}}\left(c_{i-1}^{n+1}-2 c_{i}^{n+1}+c_{i+1}^{n+1}\right) \\
= & \left(-b_{n}\right)\left(c_{i-1}^{-1}+4 c_{i}^{-1}+c_{i-1}^{-1}\right)+\left(2 b_{n}-b_{n-1}\right)\left(c_{i-1}^{0}+4 c_{i}^{0}+c_{i-1}^{0}\right) \\
& +\sum_{j=1}^{n-1}\left(-b_{j-1}+2 b_{j}-b_{j+1}\right)\left(c_{i-1}^{n-j}+4 c_{i}^{n-j}+c_{i-1}^{n-j}\right)+\left(2 b_{0}-b_{1}\right)\left(c_{i-1}^{n}+4 c_{i}^{n}+c_{i-1}^{n}\right) \\
& -\frac{r \alpha_{0}}{2}\left(c_{i-1}^{n}+4 c_{i}^{n}+c_{i-1}^{n}\right)+\frac{\alpha_{0}}{2} \frac{6}{h^{2}}\left(c_{i-1}^{n}-2 c_{i}^{n}+c_{i+1}^{n}\right)+\frac{a \alpha_{0}}{2 \Delta t}\left(c_{i-1}^{n-1}+4 c_{i}^{n-1}+c_{i+1}^{n-1}\right)+\alpha_{0} f_{i}^{n+1} .
\end{aligned}
$$

After simplification, the above equation represents the linear system of $\mathrm{N}+1$ equations in $\mathrm{N}+3$ unknowns $\mathrm{c}_{-1}^{\mathrm{n}+1}, \mathrm{c}_{0}^{\mathrm{n}+1}, \mathrm{c}_{1}^{\mathrm{n}+1}, \ldots, \mathrm{c}_{\mathrm{N}}^{\mathrm{n}+1}, \mathrm{c}_{\mathrm{N}+1}^{\mathrm{n}+1}$.

$$
\begin{aligned}
\left(1+\frac{a \alpha_{0}}{2 \Delta t}+\right. & \left.\frac{r \alpha_{0}}{2}-\frac{\alpha_{0}}{2} \frac{6}{h^{2}}\right) c_{i-1}^{n+1}+\left(4+\frac{4 a \alpha_{0}}{2 \Delta t}+\frac{4 r \alpha_{0}}{2}+\frac{6 \alpha_{0}}{h^{2}}\right) c_{i}^{n+1} \\
& +\left(1+\frac{a \alpha_{0}}{2 \Delta t}+\frac{r \alpha_{0}}{2}-\frac{\alpha_{0}}{2} \frac{6}{h^{2}}\right) c_{i+1}^{n+1}=F_{i}, \quad n \geqslant 1, \quad i=0,1,2, \ldots, N,
\end{aligned}
$$


where

$$
\begin{aligned}
F_{i}= & \left(-b_{n}\right)\left(c_{i-1}^{-1}+4 c_{i}^{-1}+c_{i+1}^{-1}\right)+\left(2 b_{n}-b_{n-1}\right)\left(c_{i-1}^{0}+4 c_{i}^{0}+c_{i+1}^{0}\right) \\
& +\sum_{j=1}^{n-1}\left(-b_{j-1}+2 b_{j}-b_{j+1}\right)\left(c_{i-1}^{n-j}+4 c_{i}^{n-j}+c_{i+1}^{n-j}\right)+\left(2 b_{0}-b_{1}\right)\left(c_{i-1}^{n}+4 c_{i}^{n}+c_{i+1}^{n}\right) \\
& -\frac{r \alpha_{0}}{2}\left(c_{i-1}^{n}+4 c_{i}^{n}+c_{i+1}^{n}\right)+\frac{\alpha_{0}}{2} \frac{6}{h^{2}}\left(c_{i-1}^{n}-2 c_{i}^{n}+c_{i+1}^{n}\right)+\frac{a \alpha_{0}}{2 \Delta t}\left(c_{i-1}^{n-1}+4 c_{i}^{n-1}+c_{i+1}^{n-1}\right)+\alpha_{0} f_{i}^{n+1} .
\end{aligned}
$$

To find unique solution of this system, eliminate $c_{-1}^{n+1}$ and $c_{N+1}^{n+1}$ using boundary conditions

$$
\begin{gathered}
u(0, t)=\left(c_{-1}+4 c_{0}+c_{1}\right)=0, \\
u(L, t)=\left(c_{N-1}+4 c_{N}+c_{N+1}\right)=0, \\
c_{-1}=-4 c_{0}-c_{1}, \\
c_{N+1}=-4 c_{N}-c_{N-1} .
\end{gathered}
$$

After eliminating $c_{-1}$ and $c_{N+1}$, the system is reduced to a tri-diagonal system of $(N+1)$ linear equations in $(\mathrm{N}+1)$ unknowns. This system can be rewritten in matrix form as

$$
\mathrm{AC}^{\mathrm{n}+1}=\mathbf{F}, \quad \mathrm{n}=1,2,3, \ldots, \mathrm{K},
$$

where

$$
\mathrm{C}^{\mathrm{n}+1}=\left[\mathrm{c}_{0}^{\mathrm{n}+1}, \mathrm{c}_{1}^{\mathrm{n}+1}, \ldots, \mathrm{c}_{\mathrm{N}}^{\mathrm{n}+1}\right]^{\top}, \quad \mathrm{n}=1,2,3, \ldots, \mathrm{K} .
$$

The coefficient matrix $\mathbf{A}$ is given as below

$$
\mathbf{A}=\left[\begin{array}{cccccc}
1 & & & & & \\
x & y & x & & & \\
& x & y & x & & \\
& & \ddots & \ddots & \ddots & \\
& & & x & y & x \\
& & & & & 1
\end{array}\right]
$$

where

$$
\begin{aligned}
& x=\left(1+\frac{a \alpha_{0}}{2 \Delta t}+\frac{r \alpha_{0}}{2}-\frac{6 \alpha_{0}}{2 h^{2}}\right), \\
& y=\left(4\left(1+\frac{a \alpha_{0}}{2 \Delta t}+\frac{r \alpha_{0}}{2}\right)+\frac{6 \alpha_{0}}{h^{2}}\right) .
\end{aligned}
$$

To calculate $\mathbf{C}^{2}=\left[\mathrm{c}_{0}^{2}, \mathrm{c}_{1}^{2}, \ldots, \mathrm{c}_{\mathrm{N}}^{2}\right]^{\top}$, first we need to calculate $\mathbf{C}^{1}=\left[\mathrm{c}_{0}^{1}, \mathrm{c}_{1}^{1}, \ldots, \mathrm{c}_{\mathrm{N}}^{1}\right]^{\top} . \mathbf{C}^{1}$ is evaluated, solving (3.7), as

$$
\begin{aligned}
(1+ & \left.\frac{\mathrm{a} \alpha_{0}}{2 \Delta \mathrm{t}}+\frac{\mathrm{r} \alpha_{0}}{2}-\frac{\alpha_{0}}{2} \frac{6}{\mathrm{~h}^{2}}\right) \mathrm{c}_{\mathrm{i}-1}^{1}+\left(4+\frac{4 \mathrm{a} \alpha_{0}}{2 \Delta \mathrm{t}}+\frac{4 \mathrm{r} \alpha_{0}}{2}+\frac{6 \alpha_{0}}{\mathrm{~h}^{2}}\right) \mathrm{c}_{\mathrm{i}}^{1} \\
& +\left(1+\frac{\mathrm{a} \alpha_{0}}{2 \Delta \mathrm{t}}+\frac{\mathrm{r} \alpha_{0}}{2}-\frac{\alpha_{0}}{2} \frac{6}{\mathrm{~h}^{2}}\right) \mathrm{c}_{\mathrm{i}+1}^{1}=\mathrm{F}_{\mathrm{i}}
\end{aligned}
$$

where

$$
\begin{aligned}
F_{i}= & \left(-1+\frac{a \alpha_{0}}{2 \Delta t}\right)\left(c_{i-1}^{-1}+4 c_{i}^{-1}+c_{i+1}^{-1}\right)+\left(2-\frac{\alpha_{0} r}{2}\right)\left(c_{i-1}^{0}+4 c_{i}^{0}+c_{i+1}^{0}\right) \\
& +\frac{\alpha_{0}}{2} \frac{6}{h^{2}}\left(c_{i-1}^{0}-2 c_{i}^{0}+c_{i+1}^{0}\right)+\alpha_{0} f_{i}^{1} .
\end{aligned}
$$




\section{Numerical results}

Following three problems have been considered to check the accuracy and efficiency of the developed method. The main purpose of these examples is to verify the convergence rate of the numerical results with respect to the fractional order $\alpha$. From the following Tables 2, 3 and 4, it is clear that the numerical results are highly consistent with the theoretical results. It can be shown that the convergence rate of numerical solutions is of order 2 as $\Delta t$ approaches zero.

Example 6.1. Following is the time-fractional telegraph equation

$$
\frac{\partial^{1.50} u}{\partial t^{1.50}}+0.1 \frac{\partial u}{\partial t}+0.1 u=\frac{\partial^{2} u}{\partial x^{2}}+f(x, t), \quad x \in[0,1], \quad 0<t \leqslant T,
$$

with the initial conditions

$$
u(x, 0)=\sin \pi x, \quad x \in[0,1],
$$

and the boundary conditions

$$
u(0, t)=u(1, t)=0, \quad 0<t \leqslant T .
$$

The exact solution of the problem is

$$
u(x, t)=(t+1)^{2} \sin \pi x .
$$

The maximum norm errors for different time steps $\Delta t$ with $\mathrm{N}=50$ and the corresponding rates of convergence at $\mathrm{T}=0.1$ are presented in Table 2 . From Table 2, it is easy to see that the temporal convergence rate of the numerical results obtained by the proposed method is consistent with the theoretical results.

Table 2: Temporal convergence rate at $\mathrm{T}=0.1$.

\begin{tabular}{|c|c|c|}
\hline$\Delta t$ & $\left\|e_{K}\right\|_{\infty}$ & Rate \\
\hline 0.001 & $6.6172 \times 10^{-4}$ & \\
\hline 0.0005 & $1.7543 \times 10^{-4}$ & 1.9153 \\
\hline 0.00025 & $4.8575 \times 10^{-5}$ & 1.8526 \\
\hline 0.000125 & $1.2143 \times 10^{-5}$ & 1.9999 \\
\hline
\end{tabular}

Example 6.2. Following is the time-fractional wave equation

$$
\frac{\partial^{\alpha} u}{\partial t^{\alpha}}=\frac{\partial^{2} u}{\partial x^{2}}+f(x, t), \quad x \in[0, L], \quad 0<t \leqslant T,
$$

with the initial condition

$$
u(x, 0)=\sin \pi x, \quad x \in[0,1],
$$

and boundary conditions

$$
u(0, t)=u(1, t)=0, \quad 0<t \leqslant T .
$$

The exact solution of the problem is

$$
u(x, t)=(t+1)^{2} \sin \pi x .
$$

The Maximum norm errors for different time steps $\Delta t$ with $N=50$ and the corresponding rates of convergence at $\mathrm{T}=0.1$ are presented in Table 3 . From Table 3 , it is easy to see that the temporal convergence rate of the numerical results obtained by the proposed method is in good agreement with the theoretical estimation. 
Table 3: Temporal convergence rate at $\mathrm{T}=0.1$

\begin{tabular}{|c|c|c|}
\hline$\Delta \mathrm{t}$ & $\left\|e_{\mathrm{K}}\right\|_{2}$ & Rate \\
\hline 0.001 & $5.5124 \times 10^{-5}$ & \\
\hline 0.0005 & $1.4871 \times 10^{-5}$ & 1.8901 \\
\hline 0.00025 & $3.8277 \times 10^{-6}$ & 1.9579 \\
\hline 0.000125 & $9.5947 \times 10^{-7}$ & 1.99617 \\
\hline
\end{tabular}

Example 6.3. Following is the time-fractional linear Klein Gordon equation

$$
\frac{\partial^{\alpha} u}{\partial t^{\alpha}}+u=\frac{\partial^{2} u}{\partial x^{2}}+f(x, t), \quad x \in[0, L], \quad 0<t \leqslant T,
$$

with the initial condition

$$
u(x, 0)=\pi^{5} \sin \pi x+\frac{1}{\pi^{5}} \cos \pi x-\frac{1}{\pi^{5}} \cos 3 \pi x, \quad x \in[0,1],
$$

and boundary conditions

$$
u(0, t)=u(1, t)=0, \quad 0<t \leqslant T .
$$

The exact solution of the problem is

$$
u(x, t)=(t+1)^{2}\left(\pi^{5} \sin \pi x+\frac{1}{\pi^{5}} \cos \pi x-\frac{1}{\pi^{5}} \cos 3 \pi x\right) .
$$

The Maximum norm errors for different time steps $\Delta t$ with $N=50$ and the corresponding rates of convergence at $\mathrm{T}=0.1$ are presented in Table 4 . From Table 4 , it is easy to see that the temporal convergence rate of the numerical results obtained by the proposed method is in good agreement with the theoretical estimation.

Table 4: Temporal convergence rate at $\mathrm{T}=0.1$.

\begin{tabular}{|c|c|c|}
\hline$\Delta \mathrm{t}$ & $\left\|e_{K}\right\|_{\infty}$ & Rate \\
\hline 0.001 & $1.8221 \times 10^{-3}$ & \\
\hline 0.0005 & $5.6177 \times 10^{-4}$ & 1.6975 \\
\hline 0.00025 & $1.5380 \times 10^{-4}$ & 1.8689 \\
\hline 0.000125 & $3.9312 \times 10^{-5}$ & 1.96801 \\
\hline
\end{tabular}

\section{Conclusion}

The solution of time-fractional hyperbolic partial differential equation has been developed. Central difference formula and cubic B-spline are used for discretizing time and space variables respectively. The proposed method is stable and the approximate results approach the analytical results with order $\mathrm{O}\left(\Delta \mathrm{t}^{2}\right)$. The approximate results obtained by the suggested method support the theoretical estimates.

\section{References}

[1] A. Atangana, Convergence and stability analysis of a novel iteration method for fractional biological population equation, Neural Comput. Appl., 25 (2014), 1021-1030. 1

[2] A. Atangana, On the stability and convergence of the time-fractional variable order telegraph equation, J. Comput. Phys., 293 (2015), 104-114.

[3] A. Atangana, On the stability of iteration methods for special solution of time-fractional generalized nonlinear ZK-BBM equation, J. Vib. Control, 22 (2016), 1769-1776. 1 
[4] A. Atangana, T. Tuluce Demiray, H. Bulut, Modelling the nonlinear wave motion within the scope of the fractional calculus, Abstr. Appl. Anal., 2014 (2014), 7 pages. 1

[5] H. M. Baskonus, F. B. M. Belgacem, H. Bulut, Solutions of nonlinear fractional differential equations systems through the implementation of the variational ieration method, Fractional Dynamics Book edited by Carlo Cattani, De Gruyter Open, (2015), Chap. 19. 1

[6] H. M. Baskonus, H. Bulut, On the numerical solutions of some fractional ordinary differential equations by fractional Adams-Bashforth-Moulton method, Open Math., 13 (2015), 547-556. 1

[7] H. M. Baskonus, H. Bulut, On the complex analytical solutions for the fractional nonlinear double sinh-Poisson equation, 1st International Symposium on Computational Mathematics and Engineering Sciences, Errichidia, Morocco, (2016). 1

[8] H. M. Baskonus, T. Mekkaoui, Z. Hammouch, H. Bulut, Active control of a chaotic fractional order economic system, Entropy, 17 (2015), 5771-5783. 1

[9] H. Bulut, H. M. Baskonus, F. B. M. Belgacem, The analytical solution of some fractional ordinary differential equations by the Sumudu transform method, Abstr. Appl. Anal., 2013 (2013), 6 pages. 1

[10] H. Bulut, H. M. Baskonus, Y. Pandir, The modified trial equation method for fractional wave equation and time fractional generalized Burgers equation, Abstr. Appl. Anal., 2013 (2013), 8 pages. 1

[11] H. Bulut, F. B. M. Belgacem, H. M. Baskonus, Some new analytical solutions for the nonlinear time-fractional KdVBurgers-Kuramoto equation, Adv. Math. Stat. Sci., 2 (2015), 118-129. 1

[12] H. Bulut, Y. Pandir, S. T. Demiray, Exact solutions of time-fractional KdV equations by using generalized Kudryashov method, Int. J. Model. Optim., 4 (2014), 315-320. 1

[13] J. Chen, F. Liu, V. Anh, S. Shen, Q. Liu, C. Liao, The analytical solution and numerical solution of the fractional diffusion-wave equation with damping, Appl. Math. Comput., 219 (2012), 1737-1748. 1

[14] H.-F. Ding, C.-P. Li, Numerical algorithms for the fractional diffusion-wave equation with reaction term, Abstr. Appl. Anal., 2013 (2013), 15 pages. 1

[15] W. Li, X. Da, Finite central difference/finite element approximations for parabolic integro-differential equations, Computing, 90 (2010), 89-111. 1

[16] C.-P. Li, Z.-G. Zhao, Y.-Q. Chen, Numerical approximation of nonlinear fractional differential equations with subdiffusion and superdiffusion, Comput. Math. Appl., 62 (2011), 855-875. 1

[17] Y. Lin, C.-J. Xu, Finite difference/spectral approximations for the time-fractional diffusion equation, J. Comput. Phys., 225 (2007), 1533-1552. 1

[18] F. Liu, V. V. Anh, I. Turner, P. Zhuang, Time fractional advection-dispersion equation, J. Appl. Math. Comput., 13 (2003), 233-245. 1

[19] F. Liu, P. Zhuang, V. Anh, I. Turner, K. Burrage, Stability and convergence of the difference methods for the space-time fractional advection-diffusion equation, Appl. Math. Comput., 191 (2007), 12-20. 1

[20] V. E. Lynch, B. A. Carreras, D. del-Castillo-Negrete, K. M. Ferreira-Mejias, H. R. Hicks, Numerical methods for the solution of partial differential equations of fractional order, J. Comput. Phys., 192 (2003), 406-421. 1

[21] M. M. Meerschaert, C. Tadjeran, Finite difference approximations for fractional advection-dispersion flow equations, J. Comput. Appl. Math., 172 (2004), 65-77. 1

[22] S. S. Siddiqi, S. Arshed, Numerical Solution of Convection-Diffusion Integro-Differential Equations with a Weakly Singular Kernel, J. Basic. Appl. Sci. Res., 3 (2013), 106-120. 1

[23] E. Sousa, How to approximate the fractional derivative of order $1<\alpha \leqslant 2$, Internat. J. Bifur. Chaos Appl. Sci. Engrg., 22 (2012), 13 pages. 1,3

[24] V. K. Srivastava, M. K. Awasthi, M. Tamsir, RDTM solution of Caputo time fractional-order hyperbolic telegraph equation, AIP Adv., 3 (2013), 11 pages. 1

[25] N. H. Sweilam, T. A. Rahman Assiri, Numerical simulations for the space-time variable order nonlinear fractional wave equation, J. Appl. Math., 2013 (2013), 7 pages. 1

[26] C. Tadjeran, M. M. Meerschaert, H. P. Scheffler, A second-order accurate numerical approximation for the fractional diffusion equation, J. Comput. Phys., 213 (2006), 205-213. 1

[27] S. Tuluce Demiray, Y. Pandir, H. Bulut, Generalized Kudryashov method for time-fractional differential equations, Abstr. Appl. Anal., 2014 (2014), 13 pages. 1

[28] L.-L. Wei, H. Dai, D.-L. Zhang, Z.-Y. Si, Fully discrete local discontinuous Galerkin method for solving the fractional telegraph equation, Calcolo, 51 (2014), 175-192. 1

[29] Y. Zhang, A finite difference method for fractional partial differential equation, Appl. Math. Comput., 215 (2009), $524-$ 529. 1

[30] H.-X. Zhang, X. Han, Quasi-wavelet method for time-dependent fractional partial differential equation, Int. J. Comput. Math., 90 (2013), 2491-2507. 1 